\title{
DNA Methylation in an Enhancer Region of the FADS Cluster Is Associated with FADS Activity in Human Liver
}

\author{
Timothy D. Howard ${ }^{1 *}$, Rasika A. Mathias ${ }^{2}$, Michael C. Seeds ${ }^{3,9}$, David M. Herrington ${ }^{4}$, James E. Hixson ${ }^{5}$, \\ Lawrence C. Shimmin ${ }^{5}$, Greg A. Hawkins ${ }^{1}$, Matthew Sellers ${ }^{4}$, Hannah C. Ainsworth ${ }^{6}$, Susan Sergeant ${ }^{7,9}$, \\ Leslie R. Miller ${ }^{8}$, Floyd H. Chilton ${ }^{8,9}$
}

1 Center for Genomics \& Personalized Medicine Research, Center for Public Health Genomics, Wake Forest School of Medicine, Winston-Salem, North Carolina, United States of America, 2 Division of Allergy and Clinical Immunology, Department of Medicine, The Johns Hopkins University, Baltimore, Maryland, United States of America, 3 Department of Internal Medicine Section on Molecular Medicine and Section on Pulmonary, Critical Care, and Allergic and Immunologic Disease, Wake Forest School of Medicine, Winston-Salem, North Carolina, United States of America, 4 Department of Cardiology, Wake Forest School of Medicine, Winston-Salem, North Carolina, United States of America, $\mathbf{5}$ Human Genetics Center, University of Texas Health Science Center at Houston, Houston, Texas, United States of America, $\mathbf{6}$ Program in Molecular Genetics and Genomics, Wake Forest School of Medicine, Winston-Salem, North Carolina, United States of America, 7 Department of Biochemistry, Wake Forest Health Sciences, Winston-Salem, North Carolina, United States of America, 8 Department of Physiology/Pharmacology, Wake Forest Health Sciences, Winston-Salem, North Carolina, United States of America, 9 Wake Forest Center for Botanical Lipids and Inflammatory Disease Prevention, Wake Forest University Health Sciences, WinstonSalem, North Carolina, United States of America

\begin{abstract}
Levels of omega-6 (n-6) and omega-3 (n-3), long chain polyunsaturated fatty acids (LCPUFAs) such as arachidonic acid (AA; 20:4, n-6), eicosapentaenoic acid (EPA; 20:5, n-3) and docosahexaenoic acid (DHA; 22:6, n-3) impact a wide range of biological activities, including immune signaling, inflammation, and brain development and function. Two desaturase steps $(\triangle 6$, encoded by FADS2 and $\triangle 5$, encoded by FADS1) are rate limiting in the conversion of dietary essential 18 carbon PUFAs (18C-PUFAs) such as LA $(18: 2, n-6)$ to AA and $\alpha$-linolenic acid (ALA, 18:3, n-3) to EPA and DHA. GWAS and candidate gene studies have consistently identified genetic variants within FADS1 and FADS2 as determinants of desaturase efficiencies and levels of LCPUFAs in circulating, cellular and breast milk lipids. Importantly, these same variants are documented determinants of important cardiovascular disease risk factors (total, LDL, and HDL cholesterol, triglycerides, CRP and proinflammatory eicosanoids). FADS1 and FADS2 lie head-to-head ( $5^{\prime}$ to $5^{\prime}$ ) in a cluster configuration on chromosome 11 (11q12.2). There is considerable linkage disequilibrium (LD) in this region, where multiple SNPs display association with LCPUFA levels. For instance, rs174537, located $\sim 15 \mathrm{~kb}$ downstream of FADS1, is associated with both FADS1 desaturase activity and with circulating AA levels ( $p$-value for AA levels $=5.95 \times 10^{-46}$ ) in humans. To determine if DNA methylation variation impacts FADS activities, we performed genome-wide allele-specific methylation (ASM) with rs174537 in 144 human liver samples. This approach identified highly significant ASM with CpG sites between FADS1 and FADS2 in a putative enhancer signature region, leading to the hypothesis that the phenotypic associations of rs174537 are likely due to methylation differences. In support of this hypothesis, methylation levels of the most significant probe were strongly associated with FADS1 and, to a lesser degree, FADS2 activities.
\end{abstract}

Citation: Howard TD, Mathias RA, Seeds MC, Herrington DM, Hixson JE, et al. (2014) DNA Methylation in an Enhancer Region of the FADS Cluster Is Associated with FADS Activity in Human Liver. PLoS ONE 9(5): e97510. doi:10.1371/journal.pone.0097510

Editor: Ludmila Prokunina-Olsson, National Cancer Institute, National Institutes of Health, United States of America

Received October 24, 2013; Accepted April 17, 2014; Published May 19, 2014

Copyright: ( $) 2014$ Howard et al. This is an open-access article distributed under the terms of the Creative Commons Attribution License, which permits unrestricted use, distribution, and reproduction in any medium, provided the original author and source are credited.

Funding: This work was supported by a grant from the National Institutes of Health, P50 AT002782. The funders had no role in study design, data collection and analysis, decision to publish, or preparation of the manuscript.

Competing Interests: The authors have declared that no competing interests exist.

*E-mail: tdhoward@wakehealth.edu

\section{Introduction}

Association studies have identified hundreds of relationships between single nucleotide polymorphisms (SNPs) and phenotypic traits [1]. However, only a few studies have clearly determined the causal mechanisms that underlie observed SNP-trait associations. Variants may affect gene expression in several ways, including altering the regulatory landscape (e.g., promoter or enhancer) of a gene, alternative splicing, transcript degradation, and transcription of non-coding RNA. However, associations are typically due to a genetic variant in linkage disequilibrium $(\mathrm{LD})$ with the associated SNP. Alternatively, an epigenetic alteration may be responsible for the observed biological variation. Recent genome-scale mapping of DNA methylation suggests that methylation impacts gene transcription via a variety of mechanisms, depending on its location (i.e., transcriptional start sites, gene bodies, regulatory elements and repeat sequences) within the transcriptional unit [2].

Long chain PUFAs (LcPUFAs) can be synthesized from essential, 18 carbon PUFAs (18C-PUFAs) found in the human diet, primarily as vegetable oil products (soybean, corn, palm, and canola oils as well as margarine and shortenings) (Figure 1). There has been a dramatic (3-4 fold) increase in the dietary levels of the omega-6 18C-PUFA, linoleic acid (to 6-8\% of daily energy consumed) in the past 50 years, as well as an intense debate about the health impact of this rise [3]. Much of this discussion centers on a difference in opinion with regard to the degree that humans have the capacity to convert 18C-PUFAs to LcPUFAs. Once 
LcPUFAs are formed, they are then incorporated into glycerolipids found in lipoprotein particles in cells and tissues (Figure 1). Once in cellular membranes, LcPUFAs can be liberated (typically after immunologic activation) as free fatty acids by a family of phospholipases $\left(\mathrm{PLA}_{2}\right)$, diacylglyceride, and monoacylglyceride lipases and converted to a large family of eicosanoid products (including prostaglandins, thromboxanes, leukotrienes and lipoxins) [4]. In general, these eicosanoids act like local hormones to regulate acute and chronic inflammation in numerous human diseases.

First cloned in $1999[5]$, the $\Delta 5$ (FADS1) and $\Delta 6$ (FADS2) desaturases are encoded by members of a gene family cluster (FADS1, FADS2 and FADS3) localized to a $100 \mathrm{~kb}$ region on chromosome 11 (11q12.2) [5,6]. Speculated to have arisen during human evolution by gene duplication, FADS1-3 have a high degree of sequence identity (62-70\%), almost identical intron/ exon organization [5] and are highly conserved between species. Numerous studies have identified associations between genetic variants in FADS1 and FADS2 and LcPUFA levels, as well as precursor-product desaturase activities, with little evidence for genetic loci outside of 11q12.2 (reviewed in Lattka et al., 2009 [7]). Other studies have found strong associations between variants in the FADS cluster with total, LDL, and HDL cholesterol, triglycerides, phospholipids, C-reactive protein, proinflammatory eicosanoids, and cadiovascular disease endpoints [8-13].

While much of the aforementioned research has been carried out in populations of European descent, recent evidence documents consistent effects in populations of African ancestry $[14,15]$. The extensive LD in European Americans is considerably less in African Americans, and rs174537 is the SNP with the strongest

\section{Dietary Essential PUFAs}
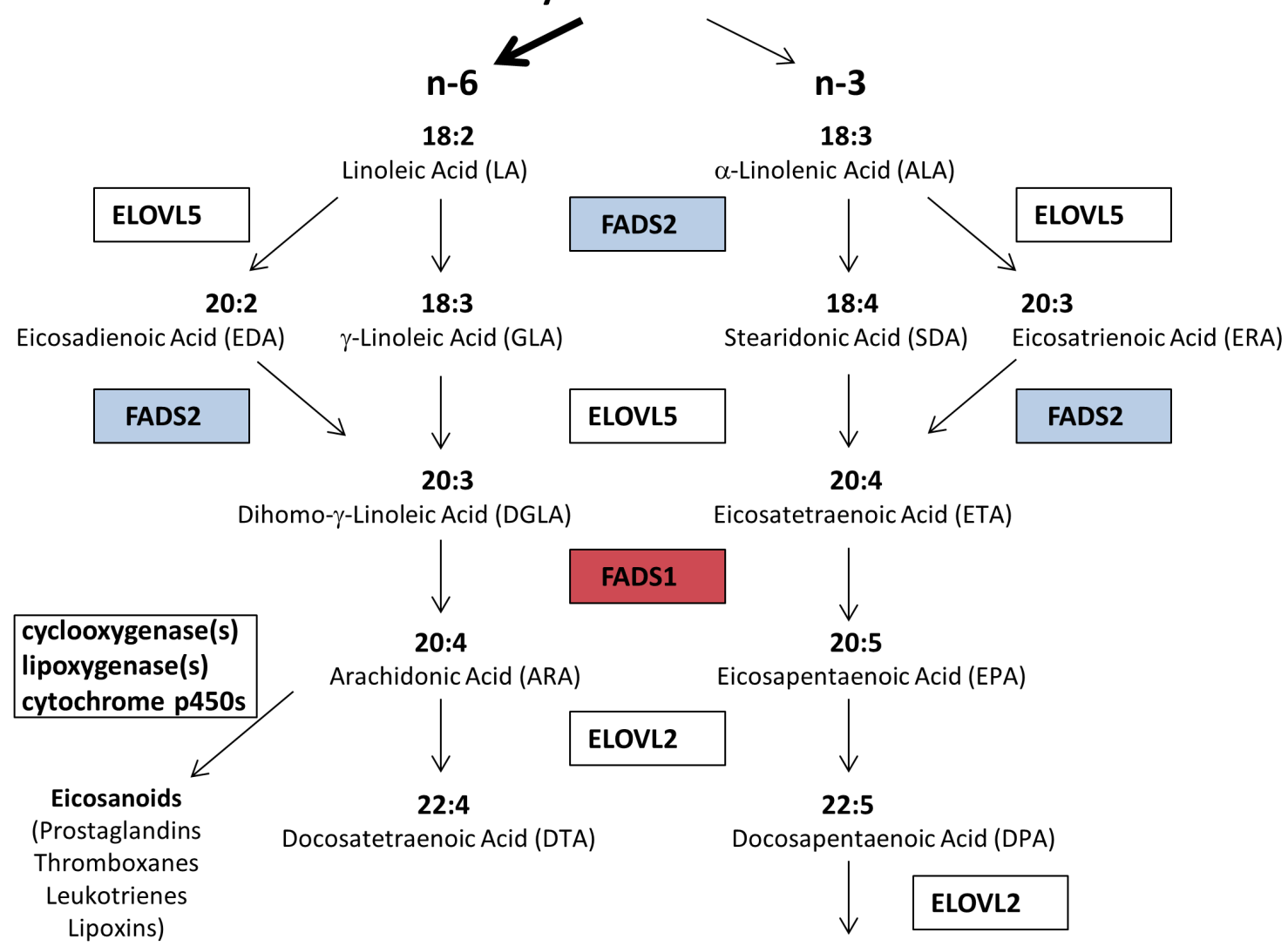

Eicosapentaenoic Acid (EPA)

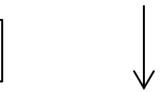

Docosapentaenoic Acid (DPA)

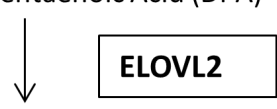

24:5

Tetracosapentaenoic Acid (TPA)

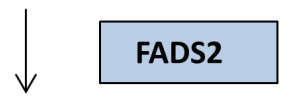

24:6

Tetracosahexaenoic Acid (THA)

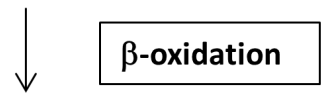

22:6

Docosahexaenoic Acid (DHA)

Figure 1. LCPUFA biosynthesis from dietary essential PUFAs Pathway. Key enzymes and metabolites are shown, and the roles of FADS1 (red) and FADS2 (blue) are indicated.

doi:10.1371/journal.pone.0097510.g001 

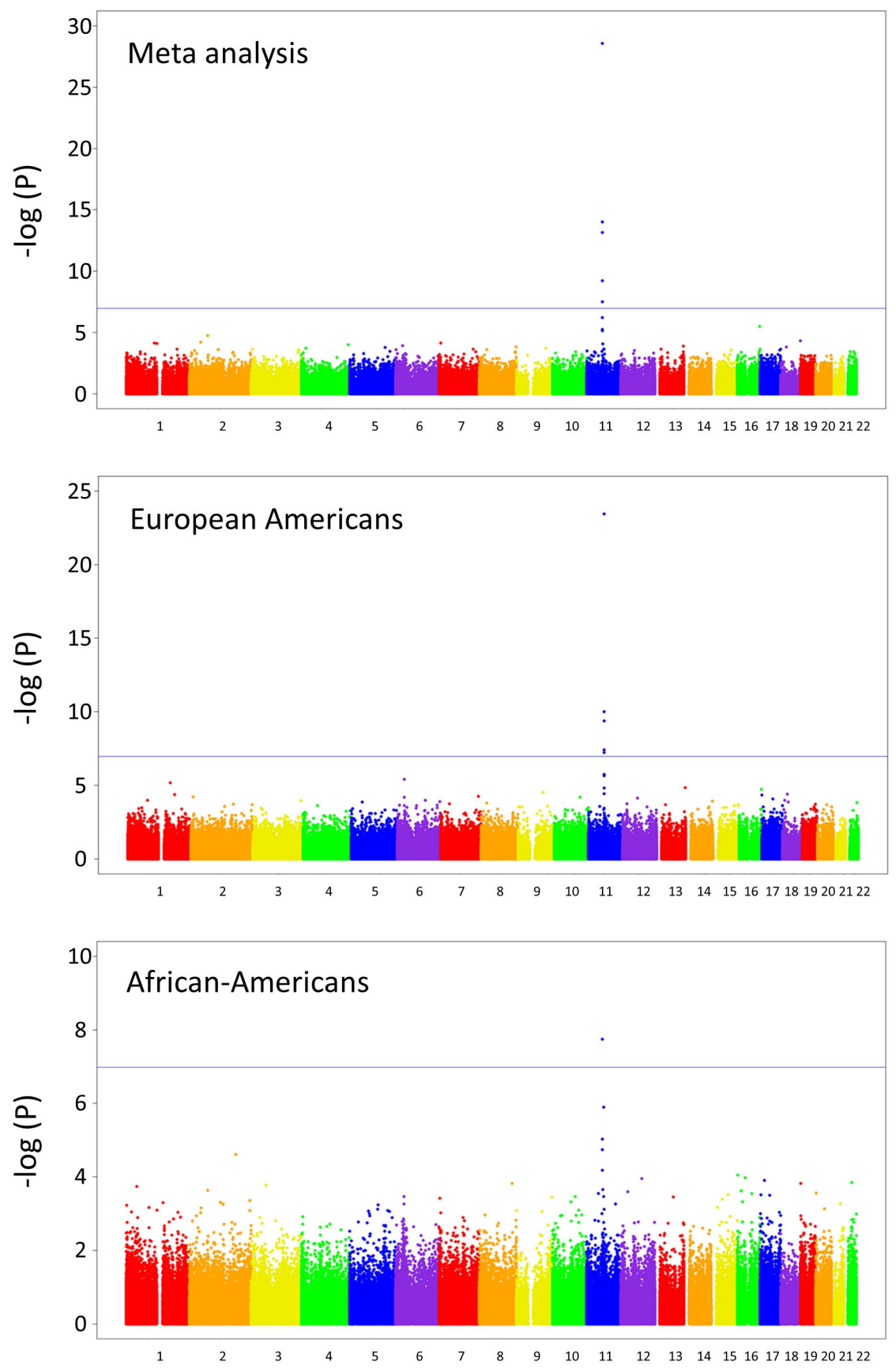

Figure 2. Manhattan plots for association of rs 174537 with HumanMethylation450 sites. P-values are based on the genetic trend test and adjusted for age, chip, and chip position. The blue line indicates the Bonferroni-adjusted level of significance of $1.03 \times 10^{-7}(0.05 / 485,000)$. The upper panel shows results from the meta-analysis (using European Americans and African-Americans), the middle panel shows European Americans only, and the lower panel shows African-Americans only. doi:10.1371/journal.pone.0097510.g002

evidence for association with AA levels [14,16]. rs174537 is located within the $3^{\prime}$ end of $M Y R F$ (myelin regulatory factor), but is also within a haplotype block spanning $\sim 30 \mathrm{~kb}$ (based on the 1000 Genomes Project) that includes FADS1 and coincides with the peak positive selection signal. This block overlaps completely with the peak association signal with LCPUFAs in African Americans [14], and includes rs174537 as well as three known eQTL SNPs for FADS1 [17] (rs174547, rs174548 and rs174549). Importantly, there are dramatic differences in the frequency of the allele $(\mathrm{G})$ associated with increased levels of LcPUFAs and FADS1 activity in African American populations [14,15] studied to date, and most notably, this allele is fixed in African populations [18]. There is strong evidence across multiple sources, including the 1000 Genomes Project and the Human Genome Diversity Panel, that this fixation is likely to have occurred as a result of positive selection approximately 85,000 years ago [18]. 


\section{Results and Discussion}

Given recent evidence that variation in DNA methylation can be associated with cis genetic variation $[19,20]$, we performed a genome-wide, allele-specific methylation (ASM) analysis with rs174537. Our hypothesis was that rs174537 may serve as a genetic proxy for DNA methylation within the FADS cluster. As liver is known to be a primary organ involved in LcPUFA biosynthesis, DNA was extracted from 144 liver samples obtained from the Pathobiological Determinants of Atherosclerosis in Youth (PDAY) study [21]. Briefly, PDAY was an autopsy study designed to examine the pathogenesis of atherosclerosis in young people. A subset of the total population, selected for a separate study to examine non-HDL cholesterol, consisted of subjects with the lowest $25^{\text {th }}$ (controls) and highest $10^{\text {th }}$ (cases) percentile of nonHDL cholesterol. Samples were from 72 European American and 72 African-American males, 15 to 34 years of age, who died of violent causes within 72 hours after injury and underwent autopsy in one of the cooperating medical examiners' laboratories. rs174537 genotypes were obtained as part of a custom genotyping panel of 77 SNPs, utilizing the Sequenom iPlex genotyping system (see Methods). To determine if ASM associations existed throughout the genome, all probes represented on the Illumina HumanMethylation450 BeadChip (485,577 CpG sites) were tested for association with rs 174537 . Only one region, on chromosome 11q12.2, showed a marked ASM association with rs174537 in both ethnicities; meta-analysis was performed combining both ethnicities, accounting for sample size and direction of effect (Figure 2). The most significant association was observed with the methylation probe $\operatorname{cg} 27386326\left(\mathrm{p}=2.69 \times 10^{-29}\right.$ for the metaanalysis; $p=3.66 \times 10^{-24}$ in European Americans and $\mathrm{p}=1.78 \times 10^{-08}$ in African Americans), located approximately $3.5 \mathrm{~kb}$ from the FADS1 transcription initiation site. As with previous association reports with rs 174537 and LcPUFA levels, the effect size of rs 174537 on methylation status was similar between the two groups $(\beta=-0.19 \pm 0.01$ in European Americans and $\beta=-0.26 \pm 0.04$ in African Americans). Four other sites $\operatorname{cg} 16213375\left(\mathrm{p}=9.76 \times 10^{-15}\right), \quad \operatorname{cg} 10515671 \quad\left(\mathrm{p}=6.93 \times 10^{-14}\right)$, $\operatorname{cg} 03805684\left(\mathrm{p}=6.22 \times 10^{-10}\right)$, and $\operatorname{cg} 19610905\left(\mathrm{p}=3.09 \times 10^{-8}\right)$ - reached a Bonferroni-adjusted level of significance (i.e., 0.05/ $485,000=1.03 \times 10^{-7}$; blue line in Figure 2). Validation of the original finding was performed using pyrosequencing of the cg27386326 CpG site, with the probe CG27386326_04 (Qiagen, Inc.). DNA methylation of the locus with pyrosequencing was strongly correlated $\left(r^{2}=0.81\right)$ with the BeadChip $450 \mathrm{k}$ result. To ensure that selection bias was not affecting our results in this population, we performed a simple association analysis with rs174537 and case-control status based on the non-HDL phenotype. No evidence of association was observed $(p=0.71$, additive model, race-adjusted).

Closer examination showed that these methylation loci are located in a likely regulatory region $5^{\prime}$ of both FADS1 and FADS2 (Figure 3). ENCODE regulation tracks revealed that the highly associated methylation region is located in a region that has a potential "enhancer signature," namely monomethylation of histone $\mathrm{H} 3$ lysine4 (H3K4mel) and acetylation of H3K27 (H3K27Ac; lower zoomed portion of Figure 3). In addition, this site is in or near transcription factor binding sites for c-Fos, STAT3 and MafK.

To better determine the putative role of cg27386326 methylation and FADS activity, we measured fatty acid levels within the liver samples and examined association between methylation levels and the key surrogate measures of FADS1 and FADS2 activities, the ratios of AA to DGLA and GLA to LA levels, respectively.
There was a strong association between methylation status of cg27386326 and AA/DGLA (FADS1 activity) in liver tissues (ANOVA $\mathrm{p}=3.99 \times 10^{-6}$; Figure 4). GLA/LA (FADS2 activity) showed a more modest relationship with methylation status $(p=0.0067$; Figure 4$)$. It is worth noting that statistical differences in associations between FADS1 and FADS2 activities may center around the difficulty of approximating FADS2 activity by measuring fatty acid levels within liver tissue. Here, the newlyformed product of the FADS2 reaction, GLA, is rapidly converted to DGLA via elongation and can no longer be measured, thus underestimating its activity. In contrast, the product of the FADS1 reaction, AA, accumulates in cells and tissues such as the liver.

Figure 4 also illustrates the distribution of rs 174537 genotypes among the samples, with GG, GT and TT clearly associated with different levels of methylation at cg27386326. This methylation proportion differed by $40 \%$ between homozygotes at rs 174537 (44\%, GG genotype; $84 \%$, TT genotype). To our knowledge, this magnitude of allele-specific or allele-associated methylation has not been observed outside of the imprinting and X-chromosome inactivation context.

One possible explanation for the association between the genetic variant rs174537 and cg27386326 methylation level is that a SNP in LD with rs 174537 exists at the targeted cg27386326 CpG site. Such a methylation-altering SNP, known as an mSNP, would lead to an apparent change in DNA methylation that was actually due to alternate genotypes at the CpG site [22]. To rule out this possibility, we sequenced the region encompassing the cg27386326 probe in the study population and found no SNP within the 50bp probe sequence or the targeted CpG site.

Both genotype and the related methylation were associated with FADS activities in the liver, but the effect of genotype nearly goes away once methylation is accounted for. Taken together, these data suggest that rs 174537, and SNPs in LD with rs174537, may serve as genetic markers for the methylation status of one or more critical CpG sites in the FADS gene cluster. This possibility is made more interesting by the noteworthy location of these sites, specifically in a region between the promoters for FADS1 and FADS2 and within a putative enhancer histone modification signature. These data also raise the key question of whether both genes are regulated by a single mechanism, the methylation of an enhancer region between two proximal promoters.

This study has several strengths and limitations. Given the nature of the PDAY study, we have been able to examine methylation levels in a highly relevant tissue for PUFA metabolism, and correlate those levels with a SNP that has been associated in population studies. Unfortunately, gene expression levels could not be measured, due to mRNA degradation of these autopsy samples. However, a recent study analyzing eQTL in liver samples from 427 Caucasian subjects showed that rs 174548 , which is in strong LD with rs174537 in the CEU population $\left(r^{2}=0.81\right.$ in the 1000 Genomes Project) was highly associated with two probes for FADS1 expression $\left(\mathrm{p}=1.52 \times 10^{-4}\right.$ and $\left.1.74 \times 10^{-5}\right)$ [17]. These data suggest that the impact of rs 174537 on FADS1 activity is altered via transcription, raising the important question of how methylation of this enhancer region impacts expression of genes in the FADS cluster. Certainly, recent studies reveal that enhancers located at variable distances from promoters can play a key role in regulating gene expression [2]. While they are typically in CpG-poor regions, whole-methylome analysis has shown them to have highly variable levels of methylation [23]. Moreover, several recent studies have linked the methylation status of an enhancer region and enhancer function, but the interaction between methylation and transcription factor binding is still poorly understood $[24,25]$. 


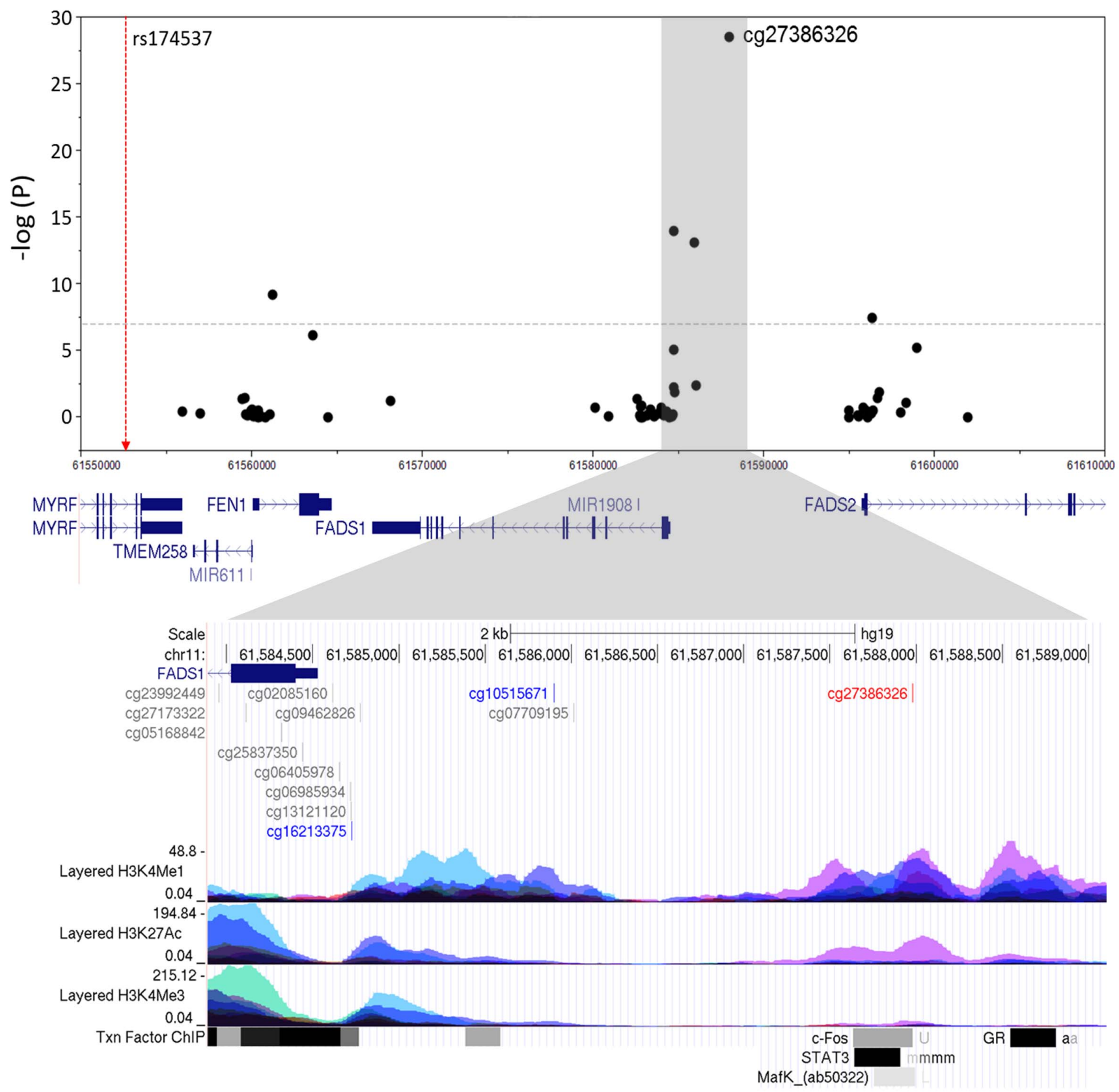

Figure 3. Association of FADS cluster CpG sites with rs174537. The lower panel shows the location of the CpG sites relative to the ENCODE data for H3K4Me1, H3K27Ac, and H3K4Me3 histone marks, respectively, which indicate promoter and/or regulatory regions in seven cell lines. The peak methylation site is shown in red, and the other two sites from the region that met Bonferroni adjustment are shown in blue. doi:10.1371/journal.pone.0097510.g003

We propose a pathway in which methylation of cg27386326 (and nearby loci) plays a key causal role in the formation of LcPUFA-containing lipids, cholesterol, triglyceride and eicosanoid formation, and ultimately clinical endpoints associated with human disease (Figure 5). As described above, there has been a dramatic increase in human exposure to n-6 18C-PUFA over the past 50 years. Additionally, it is clear that certain SNPs within the FADS cluster, such as rs174537, are strongly associated with the degree to which 18C-PUFAs are converted to LcPUFAs and the formation of LcPUFA-containing lipids [3,14,15,18]. Perhaps it is not surprising that many of these same variants are strongly associated with biomarkers of human disease, including total cholesterol, LDL-cholesterol, HDL-cholesterol, triglycerides and eicosanoids, given that LcPUFAs play a key role in the biosynthesis of each. This study suggests that rs 174537 is a strong genetic proxy for DNA methylation in a putative enhancer region of the FADS cluster, and that the methylation status of these loci may directly impact FADS1 and FADS2 activity.

\section{Materials and Methods}

\section{Study Samples}

Liver samples were obtained from the Pathobiological Determinants of Atherosclerosis in Youth (PDAY) study [21]. PDAY 

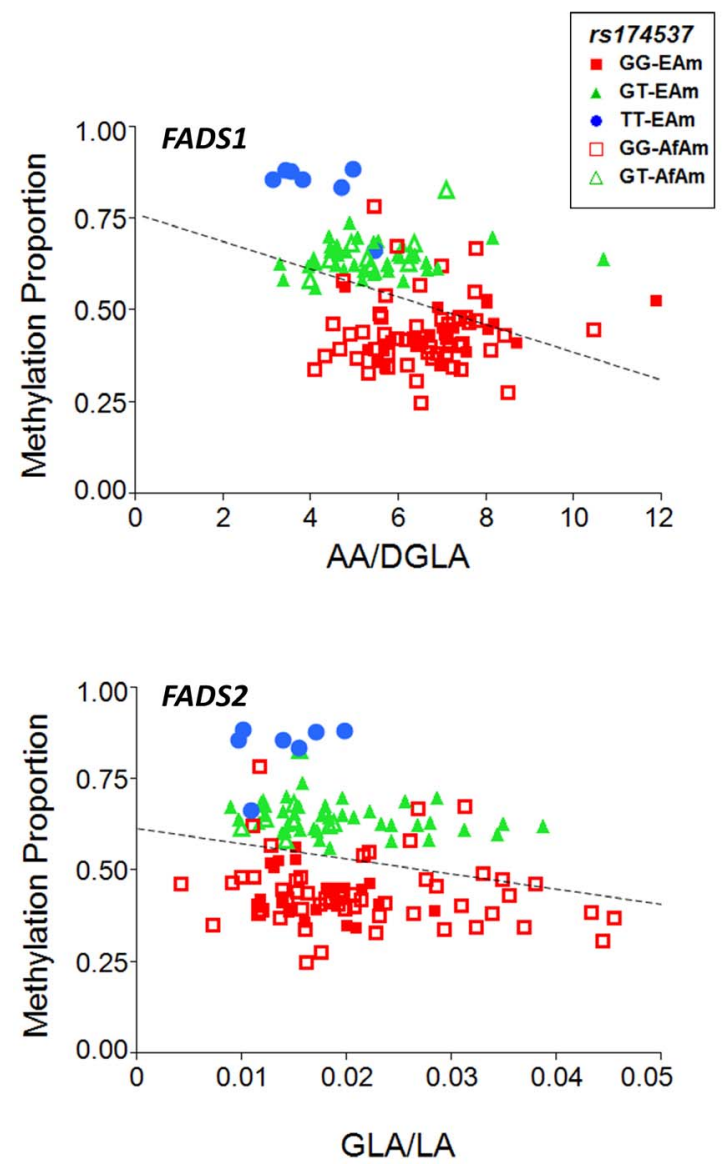

Figure 4. DNA methylation ratio and AA/DGLA (FADS1 efficiency) and GLA/LA ( $F A D S 2$ efficiency) of cg27386326 stratified by rs174537 genotype. Markers for each ethnic group, by genotype, are indicated in the legend.

doi:10.1371/journal.pone.0097510.g004

was an autopsy study designed to examine the pathogenesis of atherosclerosis in young people. A subset of the total population, selected for a separate study, consisted of subjects with the lowest $25^{\text {th }}$ (controls) and highest $10^{\text {th }}$ (cases) percentile of non-HDL cholesterol. Samples were from 72 European American and 72
African-American males, 15 to 34 years of age, who died of violent causes within 72 hours after injury and underwent autopsy in one of the cooperating medical examiners' laboratories. DNA was isolated from liver samples that had been stored at $-80 \mathrm{C}$. Five hundred to $700 \mathrm{mg}$ of thawed liver tissues were homogenized with a Dispomix Drive (Medic Tools AG, Switzerland) and genomic DNA extracted with a MagneSil Genomic, Large Volume system (Promega, USA) process that had been automated on a Freedom EVO liquid handler (Tecan, Switzerland). Extracted DNA was quantitated with PicoGreen reagent (Molecular Probes, USA) and verified as high molecular weight $(>50 \mathrm{~Kb})$ by agarose gel electrophoresis. This study used DNA obtained from liver samples acquired at autopsy. Since all study subjects were deceased at the time of study, use of these specimens is not considered Human Subjects research.

\section{SNP Genotyping}

A panel of 77 SNPs for genotyping in six fatty acid candidate genes (FADS1, FADS2, ELOVL2, ELOVL5, ACAD11 and ACOX1) were selected by using 1) using an $\mathrm{r}^{2}$ threshold of 0.7 in the HapMap CEU population, and 2) additional SNPs to guarantee tagging at an $r^{2}$ threshold of 0.7 in the HapMap YRI population, using the tagger option in Haploview [26]. rs174537 was forced to be a tagging SNP, due to its extensive associations in the literature. SNP genotyping was performed with the Sequenom iPlex genotyping system, and only rs174537 was included for analysis in this study.

\section{Sequencing Around the cg27386326 Probe Sequence}

DNA probes on the HumanMethylation450 BeadChip consist of 50bp fragments, and the subsequent base is the assayed CpG. To exclude the possibility that the CpG site included a SNP, we sequenced the 50bp probe and immediately surrounding sequence using Sanger sequencing. A 724 bp PCR product was amplified using the following primers: 5'-ATGATGTAAGTTTGGCTACAGAGA-3' and 5'-CAATTCAGCAAATTTATGTGGG-3'. PCR cycling conditions were $95^{\circ} \mathrm{C}, 5 \mathrm{~min} ; 30$ cycles of $95^{\circ} \mathrm{C}$ for $30 \mathrm{sec}, 56^{\circ} \mathrm{C}$ for $30 \mathrm{sec}$, and $72^{\circ} \mathrm{C}$ for $1 \mathrm{~min}$; followed by a $5 \mathrm{~min}$ extension at $72^{\circ} \mathrm{C}$. Sequencing reactions were performed using the ABI BigDye Terminator V1.1 chemistry. Sequencing products were run on an ABI $3730 \times$ L DNA Analyzer (Applied Bioystems, Inc., Foster City, CA) and analyzed with Sequencher V4.8 (GeneGodes Corp, Ann Arbor, MI).

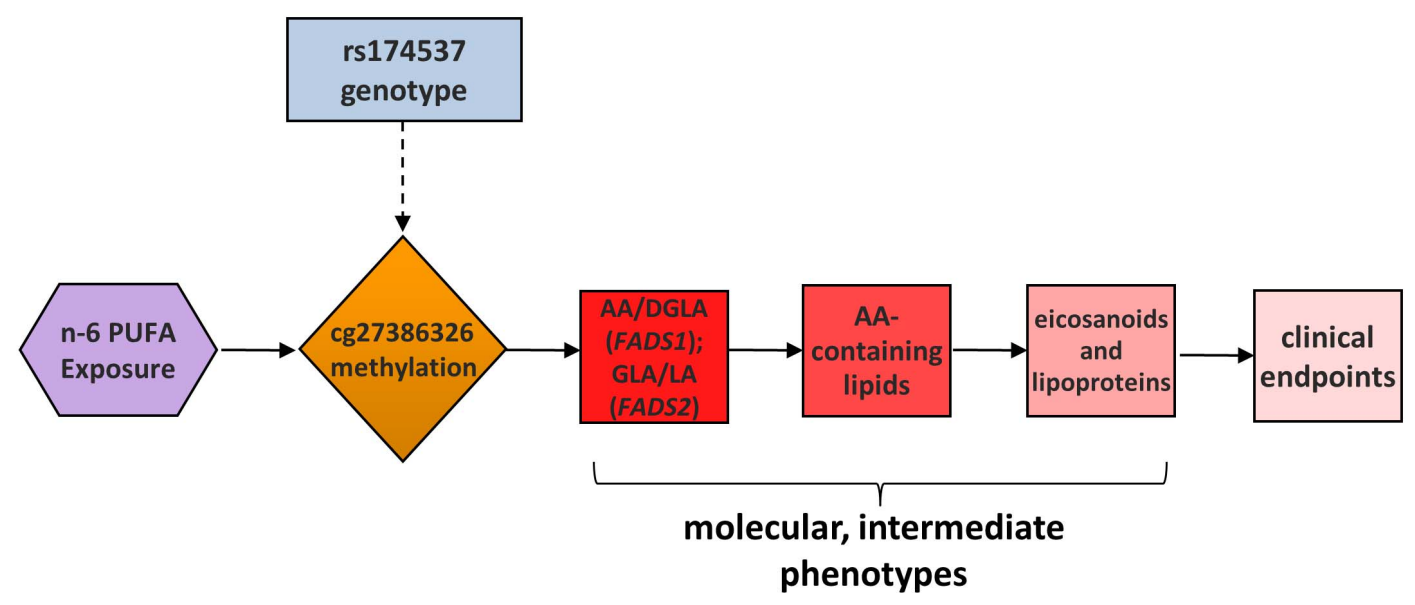

Figure 5. A model for a causal role of cg27386326 methylation in FADS activities and resulting molecular and clinical phenotypes. doi:10.1371/journal.pone.0097510.g005 


\section{DNA Methylation Analysis}

Samples were evaluated using the Illumina HumanMethylation450 BeadChip, which assays 485,577 unique CpG sites. The average beta (essentially the ratio of the methylated to unmethylated signal) for each site was used to test for differences by genotype. Association analysis was performed in the European American and African-American samples separately using a generalized linear model (proc glm), as implemented in SAS (Cary, NC). Age, chip, and chip position were included in the model as covariates. Meta analysis was performed using METAL [27], weighting by sample size and accounting for direction of effect.

\section{References}

1. Hindorff LA, Sethupathy P, Junkins HA, Ramos EM, Mehta JP, et al. (2009) Potential etiologic and functional implications of genome-wide association loci for human diseases and traits. Proc Natl Acad Sci U S A 106: 9362-9367.

2. Jones PA (2012) Functions of DNA methylation: islands, start sites, gene bodies and beyond. Nat Rev Genet 13: 484-492.

3. Blasbalg TL, Hibbeln JR, Ramsden CE, Majchrzak SF, Rawlings RR (2011) Changes in consumption of omega- 3 and omega- 6 fatty acids in the United States during the 20th century. Am J Clin Nutr 93: 950-962.

4. Needleman P, Turk J, Jakschik BA, Morrison AR, Lefkowith JB (1986) Arachidonic acid metabolism. Annu Rev Biochem 55: 69-102.

5. Marquardt A, Stohr H, White K, Weber BH (2000) cDNA cloning, genomic structure, and chromosomal localization of three members of the human fatty acid desaturase family. Genomics 66: 175-183.

6. Blanchard H, Legrand P, Pedrono F (2011) Fatty Acid Desaturase 3 (Fads3) is a singular member of the Fads cluster. Biochimie 93: 87-90.

7. Lattka E, Illig T, Heinrich J, Koletzko B (2009) FADS gene cluster polymorphisms: important modulators of fatty acid levels and their impact on atopic diseases. J Nutrigenet Nutrigenomics 2: 119-128.

8. Gieger C, Geistlinger L, Altmaier E, Hrabe dA, Kronenberg F, et al. (2008) Genetics meets metabolomics: a genome-wide association study of metabolite profiles in human serum. PLoS Genet 4: e1000282.

9. Illig T, Gieger C, Zhai G, Romisch-Margl W, Wang-Sattler R, et al. (2010) A genome-wide perspective of genetic variation in human metabolism. Nat Genet 42: $137-141$

10. Kathiresan S, Melander O, Guiducci C, Surti A, Burtt NP, et al. (2008) Six new loci associated with blood low-density lipoprotein cholesterol, high-density lipoprotein cholesterol or triglycerides in humans. Nat Genet 40: 189-197.

11. Willer CJ, Sanna S, Jackson AU, Scuteri A, Bonnycastle LL, et al. (2008) Newly identified loci that influence lipid concentrations and risk of coronary artery disease. Nat Genet 40: 161-169.

12. Kathiresan S, Willer CJ, Peloso GM, Demissie S, Musunuru K, et al. (2009) Common variants at 30 loci contribute to polygenic dyslipidemia. Nat Genet 41 : 56-65.

13. Aulchenko YS, Ripatti S, Lindqvist I, Boomsma D, Heid IM, et al. (2009) Loci influencing lipid levels and coronary heart disease risk in 16 European population cohorts. Nat Genet 41: 47-55.

14. Mathias RA, Sergeant S, Ruczinski I, Torgerson DG, Hugenschmidt CE, et al. (2011) The impact of FADS genetic variants on omega6 polyunsaturated fatty acid metabolism in African Americans. BMC Genet 12: 50.

15. Sergeant S, Hugenschmidt CE, Rudock ME, Ziegler JT, Ivester P, et al. (2012) Differences in arachidonic acid levels and fatty acid desaturase (FADS) gene

\section{Measurement of Fatty Acids in Liver Samples}

Fatty acids were measured in homogenized liver samples. Tissue was homogenized in ice-cold deionized water at $100 \mathrm{mg} / \mathrm{ml}$. Fatty acid methyl esters were prepared in triplicate homogenate samples $(100 \mu \mathrm{l})$ following a modification of Metcalfe et al. [28] and analyzed by gas chromatography as previously described [29]. Fatty acids in samples were identified based on retention times of commercially available authentic fatty acid methyl esters.

\section{Author Contributions}

Conceived and designed the experiments: TDH RAM FHC. Performed the experiments: TDH GAH HCA MCS LRM SS LCS MS. Analyzed the data: TDH RAM DMH. Contributed reagents/materials/analysis tools: JEH LCS. Wrote the paper: TDH RAM FHC SS DMH MCS LCS HCA.

variants in African Americans and European Americans with diabetes or the metabolic syndrome. Br J Nutr 107: 547-555.

16. Tanaka T, Shen J, Abecasis GR, Kisialiou A, Ordovas JM, et al. (2009) Genome-wide association study of plasma polyunsaturated fatty acids in the InCHIANTI Study. PLoS Genet 5: e1000338.

17. Schadt EE, Molony G, Chudin E, Hao K, Yang X, et al. (2008) Mapping the genetic architecture of gene expression in human liver. PLoS Biol 6: e107.

18. Mathias RA, Fu W, Akey JM, Ainsworth HC, Torgerson DG, et al. (2012) Adaptive evolution of the FADS gene cluster within Africa. PLoS One 7: e44926.

19. Gibbs JR, van der Brug MP, Hernandez DG, Traynor BJ, Nalls MA, et al. (2010) Abundant quantitative trait loci exist for DNA methylation and gene expression in human brain. PLoS Genet 6: e1000952.

20. Zhang D, Cheng L, Badner JA, Chen C, Chen O et al, (2010) Genetic control of individual differences in gene-specific methylation in human brain. Am J Hum Genet 86: 411-419.

21. Wissler RW (1991) Update on the pathogenesis of atherosclerosis. Am J Med 91: $3 \mathrm{~S}-9 \mathrm{~S}$.

22. Sigurdsson MI, Smith AV, Bjornsson HT, Jonsson JJ (2009) HapMap methylation-associated SNPs, markers of germline DNA methylation, positively correlate with regional levels of human meiotic recombination. Genome Res 19: 581-589.

23. Lister R, Pelizzola M, Dowen RH, Hawkins RD, Hon G, et al. (2009) Human DNA methylomes at base resolution show widespread epigenomic differences. Nature 462: 315-322.

24. Schmidl C, Klug M, Boeld TJ, Andreesen R, Hoffmann P, et al. (2009) Lineagespecific DNA methylation in $\mathrm{T}$ cells correlates with histone methylation and enhancer activity. Genome Res 19: 1165-1174.

25. Wiench M, John S, Baek S, Johnson TA, Sung MH, et al. (2011) DNA methylation status predicts cell type-specific enhancer activity. EMBO J 30: 3028-3039.

26. Barrett JC, Fry B, Maller J, Daly MJ (2005) Haploview: analysis and visualization of LD and haplotype maps. Bioinformatics 21: 263-265.

27. Willer CJ, Li Y, Abecasis GR (2010) METAL: fast and efficient meta-analysis of genomewide association scans. Bioinformatics 26: 2190-2191.

28. Metcalfe LD, Schmitz AA, Pelka JR (1966) Rapid preparation of fatty acid esters from lipids for gas chromatographic analysis. Anal Chem 38: 514-515.

29. Weaver KL, Ivester P, Seeds M, Case LD, Arm JP, et al. (2009) Effect of dietary fatty acids on inflammatory gene expression in healthy humans. J Biol Chem 284: 15400-15407. 